\title{
Performances de reproduction et de production des ovins de race Boujaâd au Maroc
}

\author{
A. Chikhi ${ }^{1}$ I. Boujenane ${ }^{2 *}$
}

Mots-clés

Ovin Boujaâd - Reproduction Laine - Croissance - Engraissement Carcasse - Maroc.

\begin{abstract}
Résumé
L'étude a porté sur I'analyse de 1264 performances de reproduction, 1698 poids de toison, 1588 performances de croissance et de viabilité et 184 performances d'engraissement et d'abattage des ovins de race Boujaâd. Les données ont été collectées durant sept campagnes, de 1993-1994 à 1999-2000, au domaine expérimental Déroua de I'Inra. Les brebis ont eu en moyenne un taux de fertilité de 98 p. 100, une durée de gravidité de 151 j, des tailles de portée à la naissance et au sevrage (90 j) respectivement de 1,29 et 1,24, et des poids de portée à la naissance et au sevrage respectivement de 5,03 kg et $26,8 \mathrm{~kg}$. Le poids moyen de la toison a été de 3,47 kg. Les agneaux ont pesé $3,89 \mathrm{~kg}$ à la naissance, $10,3 \mathrm{~kg}$ à $30 \mathrm{j}$ et $21,8 \mathrm{~kg}$ à $90 \mathrm{j}$. La vitesse de croissance moyenne a été de $213 \mathrm{~g} / \mathrm{j}$ de la naissance à $30 \mathrm{j}$ et de $190 \mathrm{~g} / \mathrm{j}$ entre 30 et $90 \mathrm{j}$. La moyenne du taux de la mortalité entre la naissance et $90 \mathrm{j}$ a été de 8 p. 100. Après une durée moyenne d'engraissement de 66,4 j, les agneaux nés simples et de sexe mâle ont réalisé un gain de poids moyen quotidien de $280 \mathrm{~g}$ et un indice de consommation de 4,70 kg d'aliment par kilogramme de gain de poids. Abattus à un âge moyen de $171 \mathrm{j}$, ils ont réalisé un poids vif à l'abattage de $44,8 \mathrm{~kg}$, un poids de carcasse chaude de 22,4 kg et un rendement en carcasse de 50 p. 100. En conclusion, la race Boujaâd a des potentialités très prometteuses qu'il est possible d'exploiter pour l'augmentation de la production de viande au Maroc.
\end{abstract}

\section{INTRODUCTION}

Au Maroc, l'élevage ovin constitue un secteur très important dans l'économie nationale. En effet, il contribue pour 38 à 40 p. 100 dans l'approvisionnement du pays en viande rouge, soit 21 p. 100 de la production de viande totale. Il est rencontré dans toutes les régions du pays où parfois il constitue la source essentielle de revenus des agriculteurs. Toutefois, sa productivité est faible en

\footnotetext{
1. Domaine expérimental Déroua, Institut national de la recherche agronomique, BP 567, Béni Mellal 23000, Maroc

Tél/fax : (212) 23440006 ; e-mail : chikhi@awamia.inra.org.ma

2. Département des productions animales, Institut agronomique et vétérinaire Hassan II, BP 6202, Rabat-Instituts, 10101 Rabat, Maroc Tél/fax : 21237776420 ; e-mail : i.boujenane@iav.ac.ma

* Auteur pour la correspondance
}

raison principalement du mode de conduite qui est souvent de type extensif et de l'absence de programme cohérent d'amélioration génétique pour chaque race (1).

La race Boujaâd est considérée parmi les principales races locales marocaines. Néanmoins, ses performances potentielles sont mal connues, faute d'avoir bénéficié d'un programme de recherche visant sa caractérisation. Seule, l'Association nationale ovine et caprine (Anoc) a suivi un nombre limité d'élevages dont les résultats du contrôle de performances ne sont que partiellement exploités. Des recommandations pour l'amélioration de la conduite et de la productivité basées sur des données fiables ne peuvent donc être faites pour les élevages utilisant cette race. Par conséquent, un effort important doit être investi dans ce sens afin de déterminer ses performances. La présente étude a eu pour objectif la caractérisation de la race Boujaâd et la détermination de ses performances zootechniques en station expérimentale. 


\section{MATERIEL ET METHODES}

\section{Source des données}

Les données analysées ont été collectées dans le domaine expérimental Déroua (DED) de l'Institut National de la Recherche Agronomique de Béni Mellal. Ce domaine est localisé à $24 \mathrm{~km}$ à l'ouest de Béni Mellal, sur la route nationale $n^{\circ} 8$ allant de Béni Mellal à Souk Sebt, à une altitude de $440 \mathrm{~m}$. Il est situé dans le périmètre irrigué qui relève du domaine de l'Office régional de la mise en valeur agricole du Tadla. Le domaine est situé dans l'étage bioclimatique semi-aride. La période pluviale s'étend d'octobre à mai. La pluviométrie annuelle moyenne, calculée sur une durée de 32 ans, s'élève à $386 \mathrm{~mm}$. La température moyenne est de $18{ }^{\circ} \mathrm{C}$, variant de $-4{ }^{\circ} \mathrm{C}$ au mois de janvier à $45^{\circ} \mathrm{C}$ au mois de juillet.

\section{Répartition de la race Boujaâd et description phénotypique}

La race Boujaâd, connue dans le passé sous le nom de race Tadla, est rencontrée sur les plateaux du centre-ouest du Maroc et, plus précisément, à Kasba Tadla, Oued Zem, Khouribga et Boujaâd, d'où elle a tiré son nom actuel. L'effectif total de la race Boujaâd est de 237219 têtes, soit 1,42 p. 100 de l'effectif total des ovins (1).

La race Boujaâd est caractérisée par une assez grande taille et une tête lourde armée de cornes puissantes chez le mâle et absentes chez la femelle. Le chanfrein est droit chez la brebis, large et légèrement bombé chez le bélier. L'encolure est longue, épaisse et porte un léger fanon. Le dessus est assez large et le garrot est ressorti. Le poids de la brebis varie de 45 à $60 \mathrm{~kg}$, celui du bélier oscille entre 75 et $80 \mathrm{~kg}$. La couleur de la toison est blanche, légèrement jaunâtre, ce qui confère à la race l'appellation de race Jaune. La toison est moyennement abondante, fermée, à mèches tassées, aux brins fins et ondulés de 7 à $8 \mathrm{~cm}$ de longueur. La laine est peu jarreuse et ne descend pas au-dessous du coude et du jarret. Le poids moyen de la toison est de $1,5 \mathrm{~kg}$, variant de 1,6 à $3,5 \mathrm{~kg}(1)$.

\section{Troupeau suivi et mode de conduite}

Les données analysées sont issues du troupeau du DED qui a été constitué en 1993-94 par l'acquisition de 150 brebis auprès des éleveurs sélectionneurs du berceau de la race Boujaâd. Les brebis du troupeau avaient un rang d'agnelage qui variait de 1 à 6 avec une moyenne de 3 , un âge moyen de 51 mois, un poids moyen à la lutte de $50,9 \mathrm{~kg}$ et un poids moyen à l'agnelage de $56,8 \mathrm{~kg}$.

La lutte contrôlée a été réalisée chaque année aux mois de juin et juillet et a duré $60 \mathrm{j}$. Les brebis ont été réparties en lots de lutte de 20 à 24 brebis. Dans chaque lot, un bélier intact, préalablement peint sur sa partie abdominale, pour identifier les brebis saillies, a assuré la lutte de $17 \mathrm{~h}$ à $8 \mathrm{~h}$ le lendemain.

Le troupeau ovin du DED était en stabulation permanente. L'alimentation était essentiellement basée sur le foin de luzerne, la paille, l'orge grain, le maïs grain, la pulpe sèche de betterave, le tourteau de tournesol et un complément minéral vitaminé $(\mathrm{cmv})$. Les rations ont été distribuées de façon à satisfaire les besoins des animaux aux différents stades physiologiques (lutte, fin de gravidité, lactation). Ainsi, pendant la période de lutte, il a été distribué par brebis 250 à $300 \mathrm{~g}$ d'orge, 80 à $100 \mathrm{~g}$ de tourteau de tournesol et $10 \mathrm{~g}$ de complément minéral vitaminé. En fin de gravidité, la ration par animal est passée à $400 \mathrm{~g}$ d'orge, 100 à $120 \mathrm{~g}$ de tourteau de tournesol et 10 à $20 \mathrm{~g}$ de $\mathrm{cmv}$. Durant la période d'allaitement, ont été distribués, en plus du complément de fin de gravidité, $300 \mathrm{~g}$ de pulpe sèche de betterave, par brebis.
L'alimentation des agneaux a été exclusivement à base du lait maternel au cours du premier mois de leur vie. A partir du deuxième mois, les agneaux ont reçu ad libitum un concentré spécifique composé de 60 p. 100 d'orge, 38 p. 100 du tourteau de tournesol et 2 p. $100 \mathrm{du} \mathrm{cmv}$. Sa valeur nutritive a été de l'ordre de 0,8 UF et de 16 à 18 p. 100 de matières azotées totales (Mat).

Les agneaux ont été sevrés à l'âge de 90 j et les mâles ont été séparés des femelles. Après sevrage, une trentaine d'agneaux mâles nés simples ont été conduits, chaque année, selon un régime accéléré pour être abattus après une durée d'engraissement de 60 à 70 j. Les autres étaient soit retenus pour la reproduction soit vendus.

Les agneaux d'engraissement étaient répartis en lots de 6 à 7 agneaux. L'engraissement proprement dit a commencé après une dizaine de jours d'adaptation. L'âge moyen des agneaux au début de l'engraissement a été de 104,5 j et leur poids moyen initial a été de $26,4 \mathrm{~kg}$. La ration distribuée a été formulée de manière à avoir une teneur en Mat de 16 p. 100. Cette ration a été composée de 30 p. 100 de foin de luzerne broyé, 52 p. 100 de maïs grain, 16 p. 100 du tourteau de tournesol et 2 p. 100 du cmv. Les animaux ont été abattus à un âge moyen de $171 \mathrm{j}$. Les vaccins et les traitements prophylactiques consistaient à prévenir l'entérotoxémie, la clavelée, la myopathie, ainsi que les problèmes de parasitisme interne et externe.

\section{Contrôles effectués}

$\mathrm{Au}$ cours de la période des naissances, les agneaux ont été identifiés par une boucle d'oreille et leur date de naissance, sexe et type de naissance ont été enregistrés. Ils ont également été pesés à la naissance et une fois tous les 15 jours jusqu'au sevrage. Par la suite, ils ont été pesés une fois par mois. Les agneaux sont restés entiers ; ils n'ont subi ni caudectomie ni castration. Quant aux brebis, elles ont été pesées à la lutte et à la mise bas. Le jour de la tonte, qui a lieu en avril ou mai, les poids bruts des toisons ont été enregistrés.

Pour apprécier les performances d'engraissement et les caractéristiques des carcasses de la race Boujaâd, une série de contrôles et d'observations ont été faits sur la trentaine d'agneaux engraissés et abattus chaque année. Les contrôles durant cette période ont été les suivants :

- triple pesée des agneaux au début et à la fin de la période de l'engraissement ;

- pesées simples tous les 15 jours durant la période d'engraissement ;

- contrôle quotidien de l'alimentation distribuée par lot (pesée des quantités distribuées et refusées) et son ajustement de telle sorte que le refus constituait 10 p. 100 du distribué.

Les contrôles effectués sur les agneaux abattus ont été les suivants :

- poids vif à l'abattage (après $18 \mathrm{~h}$ de jeûne) ;

- poids de la carcasse chaude (15 min après son obtention) ;

- poids du gras de rognon ;

- état d'engraissement évalué en utilisant les modèles photographiques de Colomer-Rocher (11). La note 1 correspondait aux carcasses très maigres, alors que la note 5 correspondait aux carcasses excessivement grasses.

\section{Variables étudiées}

Les performances de reproduction des brebis étudiées ont été la taille et le poids de la portée à la naissance, et la taille et le poids de la portée au sevrage ( 90 j) des brebis ayant agnelé, ainsi que la fertilité et la durée de gravidité. Les performances de croissance et de viabilité des agneaux étudiées ont été le poids à la naissance, à 
$30 \mathrm{j}$, à $90 \mathrm{j}$, à 6 mois et à un an, le gain moyen quotidien (gmq) entre la naissance et $30 \mathrm{j}$ et entre 30 et $90 \mathrm{j}$, ainsi que la viabilité des agneaux entre la naissance et $90 \mathrm{j}$. Les poids aux âges-types étudiés ont été obtenus par interpolation linéaire entre les différentes pesées effectuées. Les performances d'engraissement et les caractéristiques de carcasses étudiées ont été l'indice de consommation, le gmq à l'engraissement, le coût alimentaire par kilogramme de croît, le poids à l'abattage, le poids de la carcasse chaude, le rendement en carcasse, le gras de rognon et l'état d'engraissement.

\section{Analyses statistiques}

L'analyse a concerné 1264 performances de reproduction, 1588 performances de croissance et de viabilité, 184 performances d'engraissement et d'abattage et 1698 poids de toison. Ces données ont été collectées durant sept campagnes, de 1993-94 à 19992000. L'analyse a été réalisée à l'aide du logiciel SAS (18).

\section{RESULTATS ET DISCUSSION}

\section{Performances de reproduction}

Les moyennes arithmétiques, les écarts-types et les coefficients de variation des caractères de reproduction étudiés sont présentés dans le tableau I. La moyenne du taux de fertilité des brebis de race Boujaâd a été de 98 p. 100. Ce taux a été similaire à celui obtenu chez les brebis de race Sardi élevées dans les mêmes conditions d'élevage (9). En revanche, il a été supérieur aux taux enregistrés chez les brebis de race Sardi, Timahdite et Béni Guil élevées sur parcours, qui ont varié en moyenne respectivement de 85 à 92 p. 100, de 77 à 95 p. 100 et de 82 à 87 p. $100(1,3,7)$.

Les tailles de portée à la naissance et au sevrage des brebis de race Boujaâd ont été respectivement de 1,29 et 1,24. La taille de portée

\section{Tableau I}

Moyennes arithmétiques, écarts-types et coefficients de variation des performances de reproduction des brebis de race Boujaâd

\begin{tabular}{|c|c|c|c|c|}
\hline Caractère & Nb. & $\begin{array}{l}\text { Moy. } \\
\text { arith. } 1\end{array}$ & $\begin{array}{l}\text { Ecart- } \\
\text { type }\end{array}$ & $\begin{array}{c}\text { Coeff. } \\
\text { var. }^{2} \\
(\%)\end{array}$ \\
\hline Fertilité (\%) & 1264 & 98 & 13 & 13,3 \\
\hline $\begin{array}{l}\text { Durée de } \\
\text { gravidité (j) }\end{array}$ & 1236 & 151,4 & 2,1 & 1,39 \\
\hline $\begin{array}{l}\text { Taille de } \\
\text { la portée } \\
\text { à la naissance }\end{array}$ & 1236 & 1,29 & 0,45 & 34,9 \\
\hline $\begin{array}{l}\text { Taille de } \\
\text { la portée } \\
\text { au sevrage }\end{array}$ & 1236 & 1,24 & 0,43 & 34,7 \\
\hline $\begin{array}{l}\text { Poids de } \\
\text { la portée à la } \\
\text { naissance (kg) }\end{array}$ & 1236 & 5,03 & 1,48 & 29,4 \\
\hline $\begin{array}{l}\text { Poids de } \\
\text { la portée au } \\
\text { sevrage }(\mathrm{kg})\end{array}$ & 1236 & 26,9 & 7,2 & 26,8 \\
\hline
\end{tabular}

\footnotetext{
${ }^{1}$ Moyenne arithmétique

${ }^{2}$ Coefficient de variation
}

à la naissance a varié de 1 à 3 agneaux. Les portées simples ont représenté 71,3 p. 100 , les portées doubles 28,4 p. 100 et les portées triples 0,3 p. 100. Chikhi (9) a rapporté que cette taille de portée à la naissance était comparable à celle des brebis de race Sardi élevées dans les mêmes conditions, mais elle a été supérieure aux résultats obtenus chez les brebis des races Béni Guil (1,08 agneaux), Timahdite (1,05 agneaux) et Sardi (1,07 à 1,21 agneaux). Quant à la taille de portée au sevrage, elle a été supérieure à celles obtenues chez les brebis de races Sardi, Timahdite et Béni Guil pour lesquelles ont été enregistrés respectivement 0,99 à $1,19,1,06$ et 1,02 agneaux $(1,3,7,15)$.

Les moyennes des poids de la portée à la naissance et au sevrage des brebis de race Boujaâd ont été respectivement de $5,03 \mathrm{~kg}$ et $26,8 \mathrm{~kg}$. Le poids de la portée à la naissance enregistré a été supérieur à ceux obtenus chez les races Sardi $(3,80$ à 4,18 kg), Béni Guil $(3,7 \mathrm{~kg})$ et Timahdite $(3,6$ à $3,9 \mathrm{~kg})$. Le poids de portée au sevrage a été plus élevé que les poids enregistrés chez les races Sardi, Timahdite et Béni Guil, qui ont varié respectivement de 15,1 à $23,5 \mathrm{~kg}$, de 17,1 à $21,4 \mathrm{~kg}$ et de 16,0 à $18,2 \mathrm{~kg}(1,3,6,7,15,19)$.

La durée de gravidité des brebis de race Boujaâd a été en moyenne de $151,4 \mathrm{j}$. Cette valeur est comparable à celles rapportées par Boujenane (1) chez les brebis de races Sardi, Timahdite et Béni Guil.

Les différences entre les résultats obtenus dans la présente étude et ceux enregistrés par les différents auteurs sur les autres races locales peuvent être expliquées par des différences raciales, bien entendu, mais également par le mode de conduite et les conditions alimentaires dans chaque étude (alimentation des brebis durant les périodes de lutte, de gravidité et d'allaitement). Dans la présente étude, les brebis et les agneaux ont été en stabulation permanente et ils ont reçu leur alimentation à l'auge en fonction de leurs besoins. Alors que dans les autres études, l'alimentation des animaux était essentiellement basée sur les parcours où les problèmes de compétitivité et les risques d'une sous-alimentation des brebis gravides et en lactation se posaient, entraînant une répercussion négative sur la prolificité des brebis et sur la croissance pré- et post-natale des agneaux. Par conséquent, des plans alimentaires appropriés et une complémentation à l'auge sont préconisés pour améliorer la productivité au sevrage.

\section{Production de laine}

La moyenne du poids de la toison des béliers et des brebis de race Boujaâd tondus une fois par an a été de $3,47 \mathrm{~kg}$, avec un coefficient de variation de 23,0 p. 100 . Cette moyenne a été de $4,55 \mathrm{~kg}$ chez les béliers et de 3,34 $\mathrm{kg}$ chez les brebis. Ce poids de toison a été largement supérieur à ceux obtenus chez les races Sardi, Béni Guil, Timahdite et Béni Ahsen qui ont varié en moyenne de 1,73 à $2,96 \mathrm{~kg}(1,9,14)$. Cette supériorité peut être attribuée au grand format des ovins de race Boujaâd et à l'effet bénéfique de l'alimentation distribuée au DED.

\section{Performances de croissance des agneaux}

Les moyennes arithmétiques, les écarts-types et les coefficients de variation des performances de croissance et de viabilité des agneaux de race Boujaâd sont présentés dans le tableau II. Les moyennes des poids des agneaux de race Boujaâd ont été de $3,89 \mathrm{~kg}$ à la naissance, $10,3 \mathrm{~kg}$ à $30 \mathrm{j}, 21,8 \mathrm{~kg}$ à $90 \mathrm{j}, 32,9 \mathrm{~kg}$ à 6 mois et 53,3 kg à 1 an. La vitesse de croissance a été de $213 \mathrm{~g} / \mathrm{j}$ de la naissance à $30 \mathrm{j}$ et de $190 \mathrm{~g} / \mathrm{j}$ entre 30 et $90 \mathrm{j}$. Les mâles ont été légèrement plus lourds de la naissance à $90 \mathrm{j}$. Les vitesses de croissance ont été de $219 \mathrm{~g} / \mathrm{j}$ de la naissance à $30 \mathrm{j}$ et $200 \mathrm{~g} / \mathrm{j}$ de 30 à $90 \mathrm{j}$ chez les mâles et de $207 \mathrm{~g} / \mathrm{j}$ et $182 \mathrm{~g} / \mathrm{j}$ chez les femelles. La différence de poids s'est accentuée à 6 et 12 mois. 


\section{Tableau II}

Moyennes arithmétiques et coefficients de variation des performances de croissance et de viabilité des agneaux de race Boujaâd

\begin{tabular}{|c|c|c|c|c|c|c|c|c|c|}
\hline \multirow[t]{2}{*}{ Caractère } & \multicolumn{3}{|c|}{ Mâles } & \multicolumn{3}{|c|}{ Femelles } & \multicolumn{3}{|c|}{ Total } \\
\hline & $\mathrm{Nb}$. & $\begin{array}{l}\text { Moy. } \\
\text { arith. } 1\end{array}$ & $\begin{array}{c}\text { Coeff. } \\
\text { var. }^{2}(\%)\end{array}$ & $\mathrm{Nb}$. & $\begin{array}{l}\text { Moy. } \\
\text { arith. }\end{array}$ & $\begin{array}{c}\text { Coeff. } \\
\text { var. }(\%)\end{array}$ & Nb. & $\begin{array}{l}\text { Moy. } \\
\text { arith. }\end{array}$ & $\begin{array}{c}\text { Coeff. } \\
\text { var. (\%) }\end{array}$ \\
\hline Poids à la naissance (kg) & 782 & 4,00 & 16,7 & 806 & 3,79 & 16,3 & 1588 & 3,89 & 16,7 \\
\hline Poids à 30 j (kg) & 715 & 10,6 & 16,0 & 740 & 10,1 & 13,8 & 1455 & 10,3 & 14,6 \\
\hline Poids à 90 j (kg) & 710 & 22,6 & 15,0 & 738 & 21,0 & 11,9 & 1448 & 21,8 & 14,2 \\
\hline Poids à 180 j (kg) & 71 & 35,4 & 13,8 & 88 & 31,5 & 8,9 & 159 & 32,9 & 10,9 \\
\hline Poids à 360 j (kg) & 71 & 58,4 & 12,8 & 86 & 48,7 & 7,4 & 157 & 53,3 & 9,76 \\
\hline $\mathrm{Gmq}^{3} 0-30 \mathrm{j}(\mathrm{g})$ & 715 & 219 & 19,6 & 739 & 207 & 16,4 & 1454 & 213 & 18,3 \\
\hline Gmq 30-90 j (g) & 710 & 200 & 17,0 & 737 & 182 & 12,6 & 1447 & 190 & 15,8 \\
\hline Viabilité 0-90 j (\%) & 782 & 91 & 29,6 & 806 & 92 & 30,4 & 1588 & 92 & 28,3 \\
\hline
\end{tabular}

${ }^{1}$ Moyenne arithmétique

${ }^{2}$ Coefficient de variation

${ }^{3}$ Gains moyens quotidiens

Le poids à la naissance obtenu dans cette étude a été supérieur aux valeurs enregistrées sur les agneaux de races Sardi $(3,0$ à $3,6 \mathrm{~kg})$, Timahdite $(3,2$ à $3,6 \mathrm{~kg})$ et Béni Guil $(2,7$ à $3,3 \mathrm{~kg})$. Les poids à $30 \mathrm{j}$ et à $90 \mathrm{j}$ ont été supérieurs aux poids enregistrés chez les agneaux de race Sardi qui ont oscillé entre 6,9 et $9,8 \mathrm{~kg}$ à $30 \mathrm{j}$ et entre 15 et $21,2 \mathrm{~kg}$ à $90 \mathrm{j}$. Ils ont également été plus élevés que les poids à $90 \mathrm{j}$ observés chez les races Timahdite $(19,0 \mathrm{~kg})$ et Béni Guil $(15,3 \mathrm{~kg})(2,4,6,8,15,19)$. Ces poids ont été supérieurs aux résultats obtenus par Boujenane et coll. (2) chez les agneaux de race Sardi qui ont été de $22,7 \mathrm{~kg}$ pour les poids à 6 mois et de $30,3 \mathrm{~kg}$ pour les poids à 1 an. Les gmq $0-30 \mathrm{j}$ et $30-90 \mathrm{j}$ ont été supérieurs à ceux obtenus chez les races Sardi, Timahdite et Béni Guil qui ont varié en moyenne de 116 à $215 \mathrm{~g} / \mathrm{j}$ pour le gmq $0-30 \mathrm{j}$ et de 127 à $190 \mathrm{~g} / \mathrm{j}$ pour le gmq 30-90 j (2, 4, 6, 8, 15).

Le mode de conduite des animaux et les disponibilités alimentaires expliquent en grande partie les différences de poids et de gmq entre les différentes études. Quoi qu'il en soit, ces performances pondérales et de croissance témoignent du potentiel génétique élevé des agneaux de race Boujaâd.

\section{Viabilité des agneaux}

La distribution de fréquences de la mortalité des agneaux de race Boujaâd selon l'âge a montré que 86,8 p. 100 des mortalités globales entre la naissance et le sevrage se sont produites entre la naissance et $10 \mathrm{j}, 6,6 \mathrm{p} .100$ entre 10 et $30 \mathrm{j}$ et $6,6 \mathrm{p} .100$ entre 30 et $90 \mathrm{j}$. La principale cause de mortalité a été l'insuffisance du lait disponible pour les agneaux nés doubles. Ces résultats concordent avec ceux de Prud'hon et coll. (16) et Ricordeau et coll. (17) qui ont rapporté que 50 à 80 p. 100 des pertes se produisent au cours de la première semaine de la vie des agneaux.

La moyenne du taux de viabilité entre la naissance et $90 \mathrm{j}$ des agneaux de race Boujaâd a été de 92 p. 100 (tableau II). Ce taux été identique chez les mâles et les femelles, ainsi que, comme cela a été rapporté dans une étude précédente, chez les agneaux de race Sardi à la même station (9). En revanche, il a été plus élevé que ceux enregistrés chez les agneaux des races Sardi, Timahdite, Béni Guil et D'man qui ont varié en moyenne de 75 à 93 p. $100(1,2,6,14,15)$. Le faible taux de mortalité enregistré dans la présente étude peut être expliqué par l'effet bénéfique de l'alimentation sur la production laitière des brebis et donc sur la vigueur des agneaux.

\section{Performances d'engraissement et caractéristiques de carcasses}

Les moyennes arithmétiques, les écarts-types et les coefficients de variation des performances d'engraissement et des caractéristiques de carcasses sont rapportés dans le tableau III. Le gain moyen quotidien à l'engraissement des lots d'agneaux mâles de race Boujaâd a été en moyenne de $280 \mathrm{~g} / \mathrm{j}$. Cette valeur a été analogue à celle réalisée chez les agneaux de race Sardi dans la même station (283 g) (9), mais elle a été supérieure aux gmq enregistrés chez les agneaux de races Sardi, Béni Guil, Timahdite et D'man qui ont varié respectivement de 147 à $260 \mathrm{~g}$, de 142 à $215 \mathrm{~g}$, de 150 à $200 \mathrm{~g}$ et de 142 à $213 \mathrm{~g}(1,5,8,10,12)$. De même, ce résultat dépasse les gmq à l'engraissement réalisés par les agneaux issus du croisement industriel qui ont varié de 220 à $260 \mathrm{~g}$ (5).

Durant la période d'engraissement, le niveau d'ingestion des agneaux de race Boujaâd a été en moyenne de $1,30 \mathrm{~kg}$ de matière sèche (MS) par animal par jour, ce qui correspond à $75 \mathrm{~g}$ de $\mathrm{MS} / \mathrm{kg} \mathrm{P}^{0,75} / \mathrm{j}$. Leur indice de consommation a été de $4,70 \mathrm{~kg}$ de MS d'aliments/kg de gain de poids et de 4,17 UFV/kg de gain de poids. Ce niveau d'ingestion a été identique à celui enregistré par Chikhi (9) chez la race Sardi, mais inférieur à ceux réalisés par les agneaux issus de pères Ile de France et de mères Sardi, Béni Guil et Timahdite qui ont varié de 100 à $108 \mathrm{~g}$ de $\mathrm{MS} / \mathrm{kg} \mathrm{P} \mathrm{P}^{0,75} / \mathrm{j}$ (5). L'indice de consommation réalisé par les agneaux de race Boujaâd a été comparable aux valeurs de 4,87 $\mathrm{kg}$ de MS d'aliments $/ \mathrm{kg}$ de gain de poids et de $4,32 \mathrm{UFV} / \mathrm{kg}$ de gain de poids obtenues par Chikhi (9) chez la race Sardi. Toutefois, il a été inférieur à ceux trouvés chez les races Timahdite, Béni Guil et Sardi, qui ont varié en moyenne de 5,7 à 8,4 (5).

Abattus à l'âge moyen de $171 \mathrm{j}$, les agneaux de race Boujaâd ont réalisé un poids vif à l'abattage de $44,8 \mathrm{~kg}$ et un poids de carcasse chaude de $22,4 \mathrm{~kg}$. Ces poids ont été identiques à ceux enregistrés chez les agneaux de race Sardi abattus à l'âge de $168 \mathrm{j}$ (respectivement $45,5 \mathrm{~kg}$ et $22,4 \mathrm{~kg}$ ) (9). Cependant, ces valeurs ont été supérieures aux poids de carcasses rapportés chez les différentes races 
locales conduites en races pures ou en croisement avec les béliers à viande de races étrangères et qui ont été en moyenne de $32,2 \mathrm{~kg}$ pour les poids vifs à l'abattage et de $15,6 \mathrm{~kg}$ pour les poids de carcasse chaude (5).

Le rendement en carcasse des agneaux de race Boujaâd a été en moyenne de 50,0 p. 100. Ce résultat a été supérieur au rendement moyen de 48 p. 100 trouvé chez les agneaux de races Sardi, Béni Guil et Timahdite, et de 48,7 p. 100 obtenu chez les agneaux issus $\mathrm{du}$ croisement entre les races locales et les races à viande $(5,10,12)$.

Les carcasses des agneaux de race Boujaâd ont eu un score de l'état d'engraissement de 3,84. Ce score reflétait un bon état d'engraissement et indiquait que les carcasses produites correspondaient à la catégorie grasse. Il a été comparable à ceux observés chez les races Sardi, Béni Guil et Timahdite qui ont varié de 3,6 à 4,4 (5, 9).

La moyenne du poids du gras de rognon des agneaux de race Boujaâd a été de $298 \mathrm{~g}$. Cette valeur a été similaire au poids de $295 \mathrm{~g}$ observé par Chikhi (9) chez les agneaux de race Sardi, mais elle a été supérieure à celles rapportées par Boujenane (1) et Boujenane et coll. (5) chez les agneaux de races locales pures ou chez les agneaux croisés, qui ont varié de 182 à $260 \mathrm{~g}$.

Le coût alimentaire de l'engraissement des agneaux de race Boujaâd a été de 9,6 dirhams (DH) par kilogramme de gain du poids vif et de $19,2 \mathrm{DH} / \mathrm{kg}$ de gain de poids de carcasse (1 euro $\approx 10,5 \mathrm{DH})$. Ces valeurs ont été inférieures aux coûts trouvés chez la race Sardi par

\section{Tableau III}

Moyennes arithmétiques, écarts-types et coefficients de variation des performances d'engraissement et caractéristiques de carcasses des agneaux mâles de race Boujaâd

\begin{tabular}{|c|c|c|c|c|}
\hline Caractère & $\mathrm{Nb}$. & $\begin{array}{l}\text { Moy. } \\
\text { arith. } 1\end{array}$ & $\begin{array}{l}\text { Ecart- } \\
\text { type }\end{array}$ & $\begin{array}{c}\text { Coeff. } \\
\operatorname{var}^{2}(\%)\end{array}$ \\
\hline $\mathrm{Gmq}^{3}$ à & & & & \\
\hline I'engraissement (g) & 184 & 280 & 35 & 12,5 \\
\hline $\begin{array}{l}\text { Niveau d'ingestion } \\
\text { (kg MS/Animal/j) }\end{array}$ & 184 & 1,30 & 0,10 & 7,69 \\
\hline $\begin{array}{l}\text { Niveau d'ingestion } \\
\left.\text { (g MS/kg } \mathrm{P}^{0,75} / \mathrm{j}\right)\end{array}$ & 184 & 75,0 & 7,11 & 9,48 \\
\hline $\begin{array}{l}\text { Indice de consommation } \\
\text { (kg MS/kg de gain de poids) }\end{array}$ & 184 & 4,70 & 0,62 & 13,2 \\
\hline $\begin{array}{l}\text { Poids vif } \\
\text { à l'abattage (kg) }\end{array}$ & 184 & 44,8 & 3,6 & 8,04 \\
\hline $\begin{array}{l}\text { Poids de carcasse } \\
\text { chaude }(\mathrm{kg})\end{array}$ & 184 & 22,4 & 2,6 & 11,6 \\
\hline $\begin{array}{l}\text { Rendement } \\
\text { en carcasse (\%) }\end{array}$ & 184 & 50,0 & 3,6 & 7,20 \\
\hline $\begin{array}{l}\text { Score de l'état } \\
\text { d'engraissement }\end{array}$ & 184 & 3,84 & 0,44 & 11,5 \\
\hline $\begin{array}{l}\text { Poids du gras } \\
\text { rognon }(\mathrm{g})\end{array}$ & 184 & 298 & 95 & 31,9 \\
\hline $\begin{array}{l}\text { Coût alimentaire } \\
\text { (DH/kg du gain du poids) }{ }^{4}\end{array}$ & 184 & 9,90 & 1,50 & 15,1 \\
\hline
\end{tabular}

Chikhi (9), qui ont été de 10,1 DH/kg de gain de poids vif et de $20,2 \mathrm{DH} / \mathrm{kg}$ de gain de poids de carcasse. Elles ont également été inférieures aux coûts alimentaires de 21,2 et 23,2 DH/kg de gain de poids de carcasse rapportés par El Aouni (13) chez les agneaux respectivement de races Sardi et D'man.

\section{CONCLUSION}

A partir des résultats obtenus dans cette étude, il apparaît que la race Boujaâd a des potentialités de reproduction, de laine, de croissance, de viabilité, d'engraissement et des caractéristiques de carcasses très prometteuses et qu'il existe une variabilité importante qu'il serait possible d'exploiter pour des fins de sélection. Par conséquent, il serait intéressant d'exploiter les performances de la race Boujaâd pour l'amélioration de la production de viande au Maroc. Il serait intéressant de compléter cette étude par la détermination des effets non génétiques et par l'estimation des paramètres génétiques des différents caractères zootechniques afin de dégager les modalités les plus efficaces pour l'amélioration génétique de la race.

\section{Remerciements}

Les auteurs adressent leurs vifs remerciements à M. L. Haounou, technicien au domaine expérimental Déroua, pour sa collaboration technique.

\section{BIBLIOGRAPHIE}

1. BOUJENANE I., 1999. Les ressources génétiques ovines au Maroc Rabat, Maroc, Actes Editions, $136 \mathrm{p}$.

2. BOUJENANE I., BRADFORD G.E., BERGER Y.M., CHIKHI A., 1991. Genetic and environmental effects on growth to one year and viability of lambs from crossbreeding study of D'man and Sardi breeds. J. Anim. Sci., 69: 3989-3998.

3. BOUJENANE I., MHARCHI A., 1992. Estimation des paramètres génétiques et phénotypiques des performances de reproduction des brebis de race Beni Guil. Actes Inst. agron. vét. (Maroc), 12 : 5-13.

4. BOUJENANE I., MHARCHI A., 1992. Estimation des paramètres génétiques et phénotypiques des performances de croissance et de viabilité des agneaux de race Beni Guil. Actes Inst. agron. vét. (Maroc), $12: 15-22$.

5. BOUJENANE I., BERRADA D., MIHI S., JAMAI M., 1996. Performances d'engraissement et caractéristiques des carcasses des agneaux issus du croisement des brebis de races Timahdite, Sardi et Ben Guil avec des béliers de races à viande. Actes Inst. agron. vét. (Maroc), 16 : 29-38.

6. BOUJENANE I., CHAMI A., 1997. Effect of inbreeding on reproduction, weights and survival of Sardi and Beni Guil sheep. J. Anim. Breed. Genet., 114: 23-31.

7. BOURFIA M., TOUCHBERRY R.W., 1993. Diallel cross of three Moroccan breeds of sheep. II. Reproductive performance and productivity of pure breeds ewes. J. Anim. Sci., 71: 882-887.

8. BOURFIA M., TOUCHBERRY R.W., 1993. Diallel cross of three Moroccan breeds of sheep. I. Lamb growth and carcass traits. J. Anim. Sci., 71: 870-881.

9. CHIKHI A., 2000. Caractérisation de la race Sardi au Domaine Expérimental INRA Déroua : performances de reproduction et de productions. Mémoire pour l'obtention du grade d'ingénieur en chef Inra, Rabat, Maroc.

10. CHOULI A., OUKELMOUN M., 1983. Contribution à l'étude de la croissance et des carcasses des races ovines marocaines conduites en races pures et en croisement. Mémoire $3^{\mathrm{e}}$ cycle Agronomie, Institut agronomique et vétérinaire Hassan II, Rabat, Maroc.

11. COLOMER-ROCHER F., 1986. Méthode normalisée pour l'étude des caractères quantitatifs et qualitatifs des carcasses ovines, produites dans le bassin méditerranéen, en fonction des systèmes de production. Les carcasses d'agneaux et de chevreaux méditerranéens. Saragosse, Espagne, Ciheam 
12. ECHIGUER M., 1988. Croissance post-sevrage et caractères d'abattage des agneaux D'man et Béni Guil. Mémoire $3^{\mathrm{e}}$ cycle Agronomie, Institut agronomique et vétérinaire Hassan II, Rabat, Maroc.

13. EL AOUNI M., 2000. Comparaison des performances laitières des brebis et des performances d'engraissement et de carcasses des agneaux des races DS, D'man et Sardi. Mémoire $3^{\mathrm{e}}$ cycle Agronomie, Institut agronomique et vétérinaire Hassan II, Rabat, Maroc.

14. MHARCHI A., 1991. Analyse génétique des performances de reproduction et de production des ovins de race Beni Guil à la ferme de sélection ovine d'Oued Isly à Oujda. Mémoire $3^{\mathrm{e}}$ cycle Agronomie, Institut agronomique et vétérinaire Hassan II, Rabat, Maroc.

15. M'ZIAN S., 1994. Analyse génétique des performances de croissance et de reproduction de la race Sardi à la ferme de sélection Kra Kra. Mémoire $3^{\mathrm{e}}$ cycle Agronomie, Institut agronomique et vétérinaire Hassan II, Rabat, Maroc.

\section{Summary}

Chikhi A., Boujenane I. Reproduction and Production Performances of Boujaâd Breed of Sheep in Morocco

The study concerned the analysis of 1264 reproduction performances, 1698 fleece weights, 1588 growth and viability performances and 184 fattening and carcass performances of Boujaâd breed of sheep. Data were collected during seven periods, between 1993-94 and 1999-2000, at the Deroua Experimental Station of INRA. The ewes' fertility rate and gestation length were $98 \%$ and 151 days, respectively, litter size at birth and weaning (90 days) 1.29 and 1.24 respectively, and litter weights at birth and weaning 5.03 and $26.8 \mathrm{~kg}$, respectively. Fleece weight averaged $3.47 \mathrm{~kg}$. Lambs weighed $3.89 \mathrm{~kg}$ at birth, $10.3 \mathrm{~kg}$ at 30 days and $21.8 \mathrm{~kg}$ at 90 days of age. Their average daily gain was $213 \mathrm{~g}$ from birth to 30 days and $190 \mathrm{~g}$ between 30 and 90 days. The mortality rate from birth to 90 days averaged $8 \%$. After a fattening period of 66.4 days, male lambs born single exhibited an average daily gain of $280 \mathrm{~g}$ and a feed efficiency of $4.70 \mathrm{~kg}$ of feed per kilogram of weight gained. Slaughtered at 171 days of age, their slaughtering weight averaged $44.8 \mathrm{~kg}$, hot carcass weight $22.4 \mathrm{~kg}$, and dressing yield $50 \%$. It was concluded that the Boujaâd sheep breed has promising potentialities that could be developed to increase meat production in Morocco.

Key words: Boujaâd Sheep - Reproduction - Wool Growth - Fattening - Carcass - Morocco.
16. PRUD'HON M. DENOY I. DESVIGNES A 1968. Etude des résultats de six années d'élevage des brebis Mérinos d'Arles du domaine de Merle. III. La mortalité des agneaux. Ann. Zootech., 17 : 159-168.

17. RICORDEAU G., TCHAMITCHIAN L., LEFEVRE C., BRUNEL J.C. 1977. Amélioration de la productivité des brebis Berrichonnes du Cher (BC) par croisement. IV. Durée de gestation et viabilité des agneaux Berrichon, Romanov et croisés F1, F2, et F3. Ann. Génét. sél. Anim., 9 : 219-239.

18. SAS/STAT, 1988. User's Guide, version 6.03. Cary, NC, USA, SAS Institute.

19. TIJANI A., 1990. Analyse des performances de croissance et de reproduction chez les ovins de race Timahdite dans I'Ureo de Sidi Aissa et les troupeaux de sélection. Mémoire $3^{\mathrm{e}}$ cycle Agronomie, Institut agronomique et vétérinaire Hassan II, Rabat, Maroc.

Reçu le 18.04.2003, accepté le 22.01.2004

\section{Resumen}

Chikhi A., Boujenane I. Rendimiento de reproducción y de producción de los ovinos de raza Boujaâd en Marruecos

El presente estudio trató sobre el análisis de 1264 rendimientos de reproducción, 1698 pesos de vellón, 1588 rendimientos de crecimiento y de viabilidad y 184 rendimientos de engorde y de carcasa de los ovinos de raza Boujaâd. Los datos fueron recolectados durante siete campañas, de 19931994 a 1999-2000, en el sitio experimental de Deroua, del Inra. Las ovejas presentaron un promedio de tasa de fertilidad de $98 \%$, una duración de gestación de 151 días, tamaños de la camada al nacimiento y al destete (90 días) de 1,29 y 1,24 respectivamente y los pesos de la camada al nacimiento y al destete de $5,03 \mathrm{~kg}$ y $26,8 \mathrm{~kg}$ respectivamente. El peso medio del vellón fue de $3,47 \mathrm{~kg}$. Los corderos pesaron $3,89 \mathrm{~kg}$ al nacimiento, $10,3 \mathrm{~kg}$ a los 30 días y $21,8 \mathrm{~kg}$ a los 90 días. La velocidad de crecimiento fue de $213 \mathrm{~g} /$ día desde el nacimiento a los 30 días y de 190 g/día entre los 30 y 90 días. La tasa de mortalidad promedio entre el nacimiento y los 90 días fue de $8 \%$. Después de un periodo promedio de engorde de 66,4 días, los corderos machos, nacidos de partos simples presentaron un promedio de ganancia cotidiana de peso de $280 \mathrm{~g}$ y un índice de consumo de 4,70 kg de alimento por kilogramo de ganancia de peso. Fueron sacrificados a una edad promedio de 171 días, con un peso vivo en matadero de $44,8 \mathrm{~kg}$, un peso de carcasa caliente de $22,4 \mathrm{~kg}$ y un rendimiento de carcasa de $50 \%$. Se concluye que la raza Boujaâd presenta potenciales prometedores y que es posible explotarla para el aumento de la producción de carne en Marruecos.

Palabras clave: Ovino Boujaâd - Reproducción - Lana Crecimiento - Engorde - Cana animal - Marruecos. 